> Les anticorps thérapeutiques sont de plus en plus utilisés dans le traitement de différentes pathologies, dont les cancers et les maladies inflammatoires chroniques. La variabilité pharmacocinétique interindividuelle des anticorps est grande et influence la réponse clinique. Certaines caractéristiques de l'individu jouent un rôle majeur car elles modifient fortement cette pharmacocinétique, telles les dimensions corporelles, l'immunisation contre les anticorps thérapeutiques utilisés ou la masse antigénique. D'autres jouent un rôle mineur, comme l'âge, le sexe ou des polymorphismes génétiques. Cette variabilité doit être décrite avec soin pour déterminer le schéma posologique optimal. <

Depuis près de trois décennies, un nombre sans cesse croissant d'anticorps monoclonaux (AcM) thérapeutiques est disponible. Ils ont permis de révolutionner le traitement de pathologies de plus en plus variées, incluant les cancers et les maladies inflammatoires, mais aussi des maladies dans le domaine de l'hématologie, l'allergologie et des dyslipidémies.

Les anticorps thérapeutiques sont des immunoglobulines d'isotype $G(\lg G)$, protéines de masse moléculaire importante (environ $150 \mathrm{kDa}$ ), qui associent deux régions $\mathrm{Fab}$ (fragment antigen-binding) et une région Fc (fragment cristallisable). La région Fab contient trois régions hypervariables responsables de la fixation à la cible antigénique avec de grandes affinité et spécificité. La région Fc est impliquée dans le recrutement des effecteurs du système immunitaire, dont les effecteurs cellulaires exprimant les récepteurs de la région $\mathrm{Fc}(\mathrm{RF} \gamma)$, et la molécule $\mathrm{Clq}$ de la voie classique du complément. La région Fc permet également la fixation des IgG également au FcRn (neonatal $F_{c}$ receptor), un récepteur impliqué dans leur demi-vie.

Si beaucoup d'anticorps sont administrés par voie intraveineuse (iv), un nombre sans cesse croissant sont administrés par voie sous-cutanée ( $\mathrm{sc}$ ). Cette dernière permet une absorption lente de l'anticorps [1-4]. Si cette voie permet

\section{Variabilité pharmacocinétique des anticorps thérapeutiques}

David Ternant ${ }^{1}$, Stéphanie Chhun ${ }^{2}$

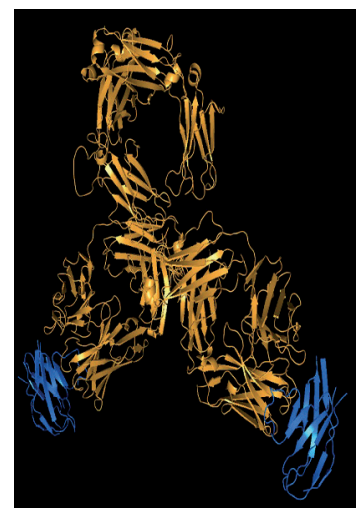

${ }^{1}$ EA 7501 GICC, Université de Tours, Tours, France ; Service de pharmacologie médicale, CHU de Tours, Tours, France. ${ }^{2}$ Hôpital Necker-Enfants Malades, Inserm U1151, INEM, Laboratoire d'immunologie biologique, Assistance PubliqueHôpitaux de Paris, Paris, France. david.ternant@univ-tours.fr

d'augmenter le confort des patients et de diminuer les coûts liés à l'hospitalisation, elle ne permet pas l'administration de grands volumes. Elle entraîne aussi un surcroît de risque immunogène et une augmentation de la variabilité pharmacocinétique (PK) [5].

L'élimination des AcM suit deux voies :

- une élimination endogène, non-spécifique, qui a lieu dans les lysosomes après endocytose. Le FcRn permet la protection des IgG de cette dégradation et leur recyclage vers le pôle vasculaire des cellules endothéliales, ce qui confère aux IgG une longue demi-vie d'élimination ( $T 12$ d'environ 3 semaines).

- une élimination impliquant la cible, spécifique, qui a lieu du fait de la fixation de forte affinité des AcM sur leurs cibles antigéniques. La probabilité de dissociation des complexes AcM-cible ainsi formés est très faible. Ils sont éliminés par internalisation pour certaines cibles exprimées à la surface cellulaire, par phagocytose (macrophages) pour les cibles circulantes, ou via d'autres cellules du système réticulo-endothélial. La cinétique d'élimination des complexes est le plus souvent plus rapide que celles de l'anticorps et de la cible à l'état libre. L'élimination des AcM est, dans certaines conditions, dépendante de la masse antigénique, qui correspond au nombre de cibles antigéniques disponibles pour être liées à l'anticorps en question. La grande spécificité de la fixation de l'anticorps à la cible n'empêche pas une grande variabilité de la réponse thérapeutique. Cette variabilité de la réponse est expliquée, au moins en partie, par la variabilité PK, qui est large chez l'homme [2-4,6]. Au 1 er janvier 2019, 177 publications indexées dans Pubmed ont analysé la PK des anticorps chez I'homme par modélisation. Parmi elles, $13 \%$ ont impliqué en première ligne le CHU et l'université de Tours et plus de $75 \%$ datent des 10 dernières années [7] $(\rightarrow)$. $(\rightarrow)$ Voir la Synthèse de G. Paintaud, $\mathrm{m} / \mathrm{s}$ $n^{\circ} 12$, décembre 2009 , page 1057 
Même si l'oncologie reste un enjeu majeur du développement des AcM, elle ne concerne que $38 \%$ des études de pharmacocinétique des anticorps chez l'homme.

La variabilité pharmacocinétique a fait l'objet de plusieurs revues, même si aucune d'elles ne présente les facteurs individuels de variabilité de façon systématique [1-3,6,8-13]. La présente revue propose une analyse systématique et synthétique des principaux facteurs influençant la variabilité PK interindividuelle des AcM.

\section{Modélisation de la pharmacocinétique des anticorps chez l'homme - État de l'art}

La PK des médicaments (en général) et des AcM (en particulier) peut être décrite de façon non-compartimentale, notamment lors des essais précoces (phase I) ou à l'aide de modèles compartimentaux (phases II-IV). La modélisation PK compartimentale permet l'estimation des paramètres PK (constantes d'absorption, de distribution et d'élimination, volumes de distribution, clairances, demi-vies) qui décrivent les différentes étapes de la PK des AcM (absorption, distribution, élimination, interaction avec la masse antigénique). Ces paramètres sont le plus souvent estimés par approche de population. II s'agit d'une approche puissante permettant non seulement la quantification de la distribution interindividuelle des paramètres PK dans la population mais, en plus, de tester et de quantifier l'association de ces paramètres avec certains facteurs individuels de variabilité (covariables) [14,15]. Puisque l'analyse de population fait l'objet de standards de qualité internationaux, elle permet également des comparaisons inter-études. La PK des anticorps thérapeutiques est le plus souvent décrite à l'aide de modèles bicompartimentaux $[1-3,6,9]$ qui en permettent une description exhaustive [16]. La valeur du volume de distribution central, de 3-4 L, permet de vérifier cette exhaustivité. La valeur du volume périphérique, de 2-4 L, montre une distribution faible mais non négligeable dans les tissus. La clairance systémique de l'anticorps est faible (0,2 à 0,4 L/jour), du fait de son recyclage par le FcRn. La cinétique de l'absorption des anticorps administrés par voie sc est invariable, décrite à l'aide d'une constante d'ordre 1, ce qui suppose une vitesse d'absorption proportionnelle à la quantité d'anticorps présent au niveau du derme (Figure 1).

La description des cinétiques conjointes de l'anticorps et de la cible est réalisée à l'aide de modèles TMDD (target-mediated drug disposition) qui ajoutent au modèle bicompartimental décrivant la cinétique de l'anticorps libre un compartiment représentant le turnover de la cible à l'état libre (son renouvellement considérant sa production et sa destruction endogènes) et un compartiment représentant l'association et la dissociation du complexe AcM-cible et la destruction de ce dernier par le système immunitaire (Figure 1) $[9,17,18]$. La masse antigénique est considérée aujourd'hui comme un facteur de variabilité majeur dont l'influence sur la PK des AcM nécessite d'être impérativement décrite de façon précise.

L'influence des covariables peut être codée de façons différentes. Pour les covariables continues (poids, concentration d'albumine, etc.), le codage est le plus souvent allométrique (loi
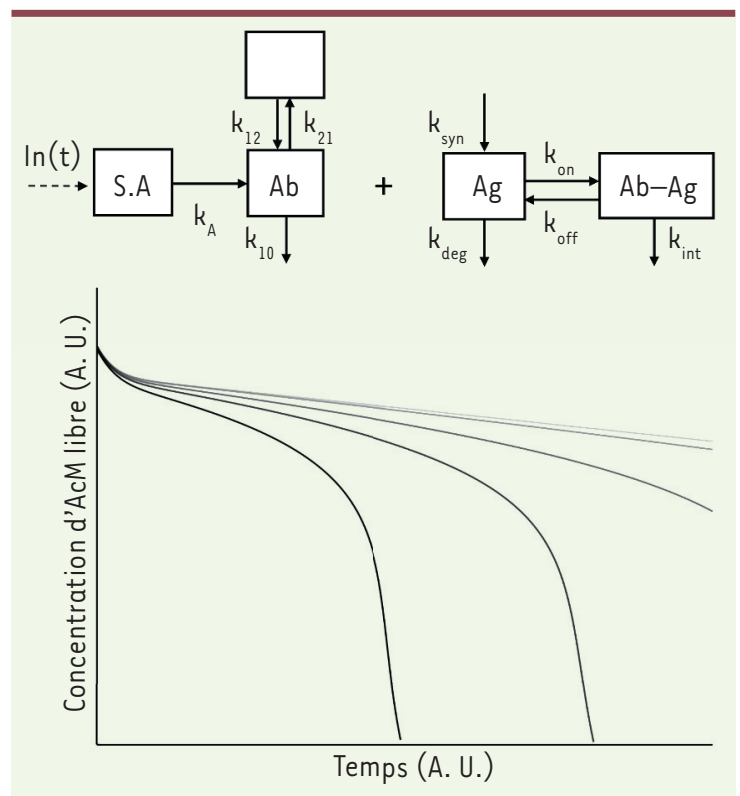

Figure 1. A. Modèle compartimental général pour décrire la pharmcocinétique des anticorps thérapeutiques. L'absorption des anticorps est décrite par une cinétique d'ordre l, c'est-à-dire une vitesse proportionnelle à la quantité d'où part le flux, quantifiée par la constante d'absorption $\mathrm{k}_{\mathrm{A}}$. La distribution vers le compartiment périphérique est bidirectionnelle, décrite par les constantes de transfert inter-compartimentales $k_{12}$ et $k_{21}$. L'élimination est décrite à l'aide de la constante d'élimination $k_{10}$. Le turnover (renouvellement) de la cible est décrit à l'aide de constantes de production $\left(k_{\text {synn }}\right.$, ordre 0$)$ et de destruction ( $k_{\text {deg }}$, ordre 1 ) endogènes. L'association et la dissociation de l'anticorps et de la cible sont décrites à l'aide des constantes respectives d'association (ordre 2) et de dissociation (ordre 1). L'élimination des complexes est décrite à l'aide de la constante $k_{\text {int }}$ (ordre 1 ), dont la valeur est le plus souvent supérieure à $k_{10}$ et à $k_{\text {deg }}$. $B$. Concentrations d'anticorps au cours du temps pour des valeurs croissantes de masse antigénique. La courbe la plus claire indique une absence de masse antigénique. Les courbes plus foncées correspondent à des masses antigéniques plus importantes, qui ont pour conséquence une non-linéarité plus prononcée.

puissance $)^{1}$, la valeur de la covariable étant rapportée à la médiane dans la population. Les covariables discrètes, notamment binaires, sont en général codées par rapport à une catégorie de référence (pour le sexe, par exemple, les femmes sont considérées comme la catégorie de référence).

\footnotetext{
${ }^{1}$ Le codage allométrique d'une covariable est réalisé à l'aide d'une loi de puissance : la relation entre le paramètre pharmacocinétique typique $\left(\theta_{T V}\right)$, c'est-à-dire celui qui quantifie la variabilité connue et la valeur de la covariable (COV) rapportée à la médiane dans la population étudiée, s'écrit : $\theta_{T V}=\left(\frac{\operatorname{cov}}{\operatorname{med}(\operatorname{COV})}\right)^{\beta_{\text {cov }}}$, où $\beta_{\text {cov est le }}$ coefficient d'association entre $\theta_{T V}$ et COV.
} 


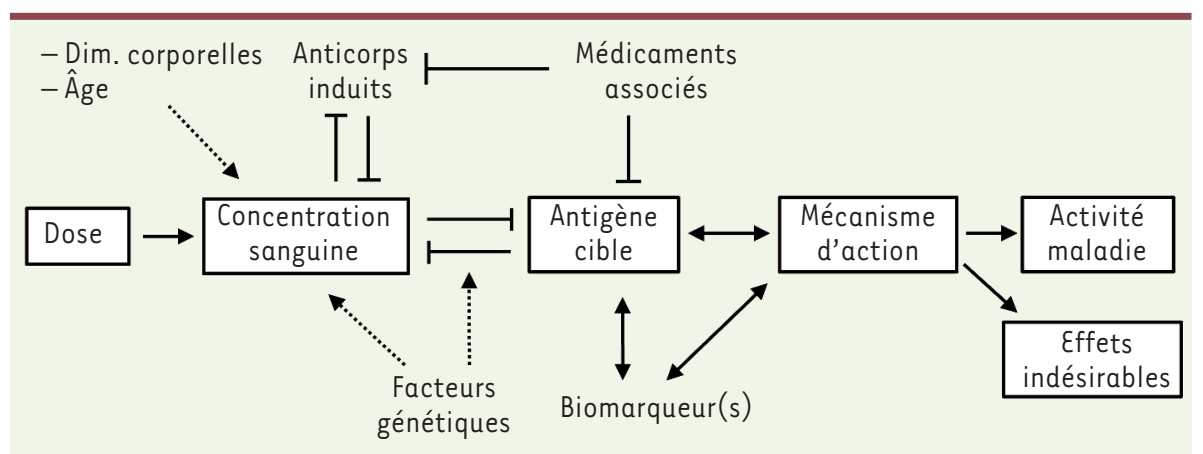

Figure 2. Schéma synthétique représentant la relation dose-concentrationeffet des anticorps thérapeutiques et les facteurs individuels de variabilité pharmacocinétique.

de l'abandon de l'ajustement de la dose aux dimensions corporelles. Indépendamment du poids, certaines études ont observé que le volume de distribution et/ou

Depuis plus de 15 ans, nous analysons de façon régulière la littérature portant sur la variabilité pharmacocinétique des anticorps chez I'homme décrite par modélisation. Parmi les 177 publications ayant modélisé la PK d'anticorps thérapeutiques chez l'homme, un modèle à 2 compartiments a été utilisé dans $80 \%$ des publications, l'utilisation d'un modèle à 1 compartiment provenait de données pauvres ou de l'absence de données PK obtenues après administration iv dans les études impliquant des AcM administrés par voie sc. L'analyse de la variabilité PK, qui est un enjeu crucial puisqu'elle influence les facteurs de variabilité PK, a été recherchée dans 137 publications.

\section{Facteurs individuels de variabilité pharmacocinétique (covariables)}

Les facteurs de variabilité dont l'influence a été la plus étudiée sont (par ordre décroissant) les dimensions corporelles (recherché dans $99 \%$ des 137 publications), l'âge (88\%), le sexe (85\%), la masse antigénique (69\%), l'origine ethnique/géographique (48\%), la concentration sérique d'albumine (50\%), les traitements concomitants (42\%) et l'immunisation (31\%). Parmi ces facteurs, ceux qui ont été retrouvés comme influençant le plus fréquemment la variabilité PK des anticorps sont (par ordre décroissant) les dimensions corporelles (influence significative dans $88 \%$ des études dans lesquelles elle a été testée), l'immunisation (74\%), la concentration d'albumine (63\%), la masse antigénique $(46 \%)$, le sexe $(33 \%)$, les traitements concomitants (26\%), l'origine ethnique/géographique (18\%) et l'âge (15\%).

\section{Influence des dimensions corporelles et du sexe}

Les dimensions corporelles sont le plus souvent mesurées à l'aide du poids corporel (dans plus de $95 \%$ des publications) et codées de façon allométrique. Des dimensions corporelles élevées sont associées à un volume de distribution et/ou une clairance élevé(s) avec un coefficient allométrique de l'ordre de 0,5-0,9. Selon les anticorps, les dimensions corporelles peuvent expliquer de 5 à $20 \%$ de la variabilité PK totale. Cette influence importante a conduit à ajuster la posologie au poids (ou à la surface corporelle), notamment pour les anticorps injectés par voie iv, cet ajustement existant de façon marginale pour les anticorps injectés par voie sc (par exemple l'omalizumab, un anticorps anti- $\mid g \varepsilon)$. La variabilité PK des anticorps injectés par voie sc est donc susceptible d'être plus importante que celle des anticorps injectés par voie iv, du fait d'un surplus de variabilité dû à l'absorption et la clairance étaient plus élevées chez les hommes (5-15\%) que chez les femmes. Cette influence n'a toutefois jamais conduit à un ajustement posologique par rapport au sexe.

\section{Influence de l'immunisation}

Dès le début des années 2000, l'utilisation des anticorps anti-TNF- $\alpha$ (tumor necrosis factor alpha) dans les maladies inflammatoires chroniques a fait l'objet de plusieurs analyses en ce qui concerne la PK. En effet, les AcM injectés peuvent induire la formation d'anticorps (ADA pour anti-drug antibodies), dont la présence est associée à des concentrations d'anticorps thérapeutiques diminuées, parfois très fortement $[4,19]$, en raison d'une augmentation de leur clairance variant d'un facteur 1,5 à 4 , et ceci même avec les anticorps « complètement » humains, comme l'adalimumab $[4,20]$. La présence d'ADA serait ainsi la principale cause de perte de réponse aux traitements par les anticorps anti-TNF- $\alpha$. À noter que l'utilisation de traitement immunosuppresseurs, dont le méthotrexate, a permis de diminuer le risque de développer des ADA chez les patients atteints de polyarthrite rhumatoïde et traités par des AcM anti-TNF- $\alpha$ [21]. Toujours dans la polyarthrite rhumatoïde, le traitement par le rituximab peut également induire le développement d'ADA, sans toutefois altérer la réponse clinique [22]. Ces anticorps sont rarement recherchés en cancérologie. Cependant, leur présence est associée à une clairance augmentée, dans $4 / 9$ des publications concernant cette question, et peut altérer la réponse clinique, comme pour l'ipilimumab (anti-CTLA-4 [cytotoxic T-lymphocyte-associated protein 4]) [23]. Il est donc indispensable que la recherche de l'influence des ADA sur la PK et la relation concentration-effet des anticorps soit systématiquement recherchée lors du développement des molécules.

\section{Influence de la concentration d'albumine sérique}

La concentration pré-thérapeutique d'albumine dans le sérum est le plus souvent retrouvée inversement corrélée à la clairance des anticorps lorsqu'elle est testée 
(avec un coefficient allométrique de l'ordre de -0,9). La grande majorité des études interprètent ce résultat de la façon suivante : puisque l'albumine et les IgG sont toutes deux recylées par le FcRn dans le sérum de façon vraisemblablement non-compétitive [24], le lien entre forte concentration d'albumine et faible clairance des anticorps pourrait être dû à une activité du FcRn élevée. Certaines études admettent toutefois que cette explication est approximative, évoquant notamment les liens étroits entre inflammation, concentration d'albumine, expression du FcRn, et même masse antigénique [25]. Même s’il n'existe pas de lien causal direct entre concentration d'albumine et PK des anticorps, elle est néanmoins considérée comme un «marqueur » de leur élimination endogène.

\section{Influence de la masse antigénique}

Comme nous l'avons évoqué, la forte affinité de la liaison entre l'anticorps et sa cible entraîne une très faible probabilité de dissociation des complexes AcM-cible. Leur élimination est donc le plus souvent plus rapide que leur dissociation. Ainsi, une quantité de cibles élevée est associée à des concentrations d'anticorps faibles, ce qui est le plus souvent dû à une clairance spécifique (reposant sur la cible) d'autant plus élevée que la masse antigénique est forte. La variabilité au cours du temps de la masse antigénique confère, dans certains cas, une variation des paramètres PK des anticorps (notamment de la clairance) et donc une PK non-linéaire, qui sera observée notamment lorsque l'anticorps sera en défaut stœchiométrique par rapport à la masse antigénique. Une PK non-linéaire est rapportée dans $38 \%$ des publications [9]. Les modèles TMDD, qui permettent de décrire les cinétiques conjointes de l'anticorps et de la cible et donc d'évaluer la clairance dépendant de la cible, nécessitent de mesurer la concentration d'anticorps, ainsi que les quantités de cibles libres et des complexes, ce qui est rarement possible. Des modèles simplifiés peuvent être utilisés pour décrire l'élimination non-linéaire des anticorps: modèles imposant une fixation anticorpscible à l'équilibre (hypothèse de quasi-steady-state, modèle qui néglige la dissociation des complexes anticorps-cible (irreversible binding), ou modèle imposant une masse antigénique totale (libre + liée) constante (modèles à élimination de Michaelis-Menten) $[9,17,18,26]$. On notera que la proportion de publications où une PK non-linéaire est rapportée concerne deux fois plus souvent la cancérologie (48\% des publications) que les autres pathologies (23\%).

Toutefois, une PK apparemment linéaire indique un excès stœchiométrique de l'anticorps et donc une saturation de l'élimination spécifique, et non une absence d'influence de la masse antigénique. En effet, la masse antigénique est décrite comme influençant significativement la PK dans $43 \%$ des études dans lesquelles la PK était apparemment linéaire. La masse antigénique peut être mesurée de façon très spécifique (quantité/concentration de la cible), moyennement spécifique (biomarqueur corrélé à la masse antigénique) ou peu spécifique (activité de la maladie). À titre d'exemple, la corrélation positive entre la concentration sérique de CRP (C-reactive protein), une protéine produite au cours des inflammations, et la clairance des anticorps anti-TNF- $\alpha$ est probablement due au fait que les concentrations de CRP et de TNF- $\alpha$ sont liées [3], I'influence de la CRP étant un reflet de l'élimination spé- cifique de ces AcM. Dans la majorité des cas, la masse antigénique a été testée en tant que covariable sur les paramètres PK et une corrélation positive entre masse antigénique et clairance de l'anticorps a été observée. Dans de rares cas, une masse antigénique élevée semble associée à une augmentation de la demi-vie terminale, comme pour le rituximab dans les lymphomes de haut grade [27], l'anticorps étant alors d'autant plus « séquestré » que la masse antigénique est importante. Cette situation s'observerait dans les cas où l'élimination des complexes est lente [9].

\section{Influence des traitements concomitants}

Puisque ni l'absorption ni l'élimination des anticorps ne font intervenir de transporteurs ni de (co)enzymes de métabolisme, il est courant de penser que les AcM ne peuvent faire l'objet d'interactions médicamenteuses [28]. Toutefois, il a été rapporté que la co-administration de méthotrexate était associée à une diminution de la clairance de l'infliximab et de l'adalimumab (deux anticorps anti-TNF- $\alpha$ ). Le méthotrexate pourrait en fait participer à la réduction des concentrations sériques de TNF- $\alpha$, et donc diminuer l'élimination spécifique et la clairance totale des anticorps spécifiques. De même, cinq publications sur les six portant sur la pharmacocinétique des AcM anti-PCSK9 (proprotéine convertase subtilisine/kexine de type 9) ont montré une influence des statines sur leur élimination, avec une clairance endogène [29] ou une élimination spécifique [30] des anticorps, augmentées en leur présence. En effet, I'utilisation des statines semble augmenter la sécrétion de la PCSK9 [28], ce qui provoque l'augmentation de la formation de complexes AcM-PCSK9, et donc l'élimination accrue de l'anticorps [31].

\section{Influence de l'âge}

L'influence de l'âge sur la PK des anticorps reste peu claire. Chez les adultes, l'augmentation de la clairance avec l'âge est la plus fréquemment rapportée. Cependant, la clairance des anticorps semble être supérieure chez les enfants comparativement à celle des adultes, et ce malgré une augmentation de la clairance avec le poids corporel. Cela a été montré pour le canakinumab (un anticorps anti-IL-1 $\beta$ ) [32]. Les raisons de cette augmentation restent obscures, même si des différences en termes de perméabilité et de densité capillaires et d'expression du FcRn ont été évoquées [33].

\section{Influence de l'origine ethnique/géographique}

Dans la mesure où règne une grande hétérogénéité dans la définition même des critères ethniques (définis sous les termes «race» ou « ethnicity» dans les 
publications) ou géographiques, ainsi que dans leur codage, il est très difficile, voire impossible, de décrire précisément cette influence. Toutefois, certaines études ont rapporté une clairance augmentée chez les sujets d'origine sub-saharienne. Les études rapportant une influence de l'origine ethnique/géographique lui reconnaissent toutefois une force insuffisante pour être prise en compte ou interprétée.

\section{Autres facteurs individuels de variabilité}

Nous présentons dans cette partie des facteurs individuels de variabilité moins fréquemment testés et/ou détectés comme influençant la PK des anticorps. Ils ne sont exposés ici que dans un but scientifique. Ils sont en effet trop inconstamment détectés et/ou présentent un effet trop faible pour être pris en compte en recherche clinique.

\section{Fonctions rénale et hépatique}

Dans les protocoles de recherche clinique, notamment industriels, il est souvent recherché une influence de la fonction rénale (créatininémie, clairance de la créatinine) et de la fonction hépatique (activité enzymatique des amino-transférases, gamma glutamyl transférases) sur la PK des anticorps, même si une telle influence n'est théoriquement pas possible. Cependant, une fonction rénale augmentée (clairance de la créatinine élevée) et/ou une fonction hépatique diminuée (enzymes augmentées) sont associées à une clairance des AcM accrue dans environ $20 \%$ des publications dans lesquelles ces associations ont été examinées. Difficiles à interpréter, ces associations sont souvent considérées comme liées à d'autres variables qui n'ont pas été analysées.

\section{Influence des IgG endogènes}

Même si elles sont rarement testées, les concentrations d'IgG endogènes sont plus d'une fois sur deux corrélées positivement à la clairance des AcM. Une interprétation possible est que des concentrations élevées d'IgG, en plus des AcM administrés, sont susceptibles d'entraîner une saturation du FcRn, provoquant une diminution de la proportion d'IgG recyclée et donc une augmentation de leur clairance, dont celle de l'anticorps thérapeutique.

Schématiquement, les chaînes lourdes des IgGl (les plus nombreuses) présentent deux allotypes qui confèrent aux immunoglobulines des affinités différentes vis-à-vis du FcRn : l'allotype Glm17,1 présentant une meilleure affinité que l'allotype Glm3,-1. Chez les patients atteints de spondylarthropathies traités par l'infliximab, d'allotype Glm17,1, la demi-vie d'élimination de l'AcM est plus élevée chez les patients dont les IgG sont d'allotype Glm3,-l que chez les autres patients. En effet, l'allotype Glm17,l ayant une affinité plus élevée pour le FcRn, l'infliximab est un meilleur compétiteur pour le FcRn que les IgG endogènes des sujets $\mathrm{Glm} 3,-1$. L'AcM est donc mieux recyclé que les immunoglobulines du patient. Ce recyclage est plus faible chez les sujets Glm17,1 pour lesquels l'infliximab et leurs IgG endogènes ont des affinités pour le FcRn identiques [34].

\section{Polymorphismes génétiques}

Le gène FCGRT (Fc fragment of IgG receptor and transporter) qui code le $\mathrm{FcRn}$, présente un nombre variable de répétions d'une séquence par- ticulière (VNTR pour various number tandem repeat). Le niveau d'expression du récepteur est lié au nombre de ces répétitions [35]. Les patients homozygotes pour 3 répétitions semblent ainsi présenter une activité de distribution et d'élimination des anticorps plus élevée [36, 37] Cet effet reste toutefois d'amplitude relativement faible et ne présente probablement pas d'intérêt clinique. Le gène FCGR3A (Fc IgG receptor II/a) code le récepteur RFcyllla, ou CDI6a, un récepteur de la région $F c$ des IgG exprimé à la surface d'effecteurs cellulaires, notamment des cellules NK. Ce gène présente un polymorphisme bi-allélique, FCGR3A-158V/F, qui génère un allèle $V$ (valine) ou $F$ (phénylalanine). In vitro, le récepteur présentant la valine à la position 158 a une affinité plus importante pour les IgG que celui présentant une phénylalanine à cette position. In vivo, chez les patients atteints de lymphomes non-Hodgkiniens folliculaires, cela se traduit par une meilleure efficacité du rituximab chez les homozygotes V/V que chez les porteurs du variant $F$. Ce polymorphisme influence la relation concentration-effet des anticorps, aussi bien ex vivo [38] qu'in vivo $[39,40]$ chez des patients transplantés rénaux traités par immunoglobulines anti-lymphocytaires. Cependant, dans la maladie de Crohn, pour l'infliximab et dans la leucémie lymphoïde chronique pour le rituximab, le génotype V/V a été associé à une élimination spécifique plus élevée que chez les patients présentant un autre polymorphisme. Cela suggère que la meilleure affinité du FcyRIIla V/V pour les IgG entraîne une élimination plus rapide des complexes anticorps-cellule par les cellules NK, et donc une plus grande «consommation» de l'anticorps thérapeutique [9].

\section{Conclusions}

La variabilité pharmacocinétique des anticorps, habituellement assez large, nécessite d'être décrite précisément car elle influence, au moins en partie, la variabilité de leur effet clinique. Aujourd'hui, un grand nombre de facteurs individuels permettent d'expliquer cette variabilité à des niveaux très divers. Si certains facteurs (dimensions corporelles, immunisation, masse antigénique, concentration d'albumine) constituent des prédicteurs ${ }^{2}$ de variabilité quantitativement pertinents, d'autres facteurs ont une influence plus modeste. Ils peuvent cependant présenter un intérêt dans la compréhension du mécanisme d'action des anticorps chez I'homme (IgG endogènes, polymorphismes génétiques). Certains facteurs, dont les dimensions corporelles ou

\footnotetext{
${ }^{2}$ Variables indépendantes ou variables d'entrée.
} 
l'âge, mériteraient des analyses plus poussées. En effet, si le poids est la mesure corporelle de loin la plus utilisée, certaines études ont montré que d'autres mesures (taille [36] ou poids maigre [41]) ont une influence plus importante sur la variabilité PK.

Le développement d'ADA a été particulièrement décrit dans le cadre des pathologies inflammatoires chroniques, notamment parce que leur présence entraîne bien souvent une augmentation de la clairance de l'anticorps qui se traduit par des concentrations très diminuées et un risque de perte de réponse secondaire à ce phénomène. Les incertitudes autour des ADA sont toutefois nombreuses. En effet, le risque de perte de réponse en présence d'ADA est très variable selon les anticorps, les pathologies et les patients, et il n'existe aujourd'hui aucune méthode fiable pour prédire le risque individuel d'immunisation et ses implications cliniques avant le traitement. Ce risque reste encore aujourd'hui un champ majeur de recherches [3].

La masse antigénique modifie la PK des anticorps, là aussi de façon variable en fonction des anticorps, des pathologies et des patients. La quantification de cette influence chez l'homme est cependant essentielle car elle détermine la première dose à administrer, qui ne peut que très difficilement être extrapolée de l'animal. Elle permettra également de définir la dose à administrer, afin que l'anticorps soit en excès stœchiométrique par rapport aux cibles chez la majorité des patients. Toutefois, cette stratégie a ses limites. Elle peut, notamment, conduire à une sur-exposition chez la majorité des patients, avec, en plus d'une augmentation du risque de survenue d'effets indésirables, un surcoût qui peut être important. L'ajustement de la dose à la quantité de masse antigénique n'est toutefois par la règle dans la mesure où celle-ci n'est pas toujours directement mesurable [9].

Même si l'influence de l'âge sur la PK des anticorps a été peu décrite, il semble exister des différences notables entre les adultes et les jeunes enfants. Ces derniers semblent en effet éliminer les anticorps de façon plus rapide que les adultes. Il est probable que le schéma posologique de certains anticorps nécessite d'être adapté aux indications pédiatriques. Si de nombreux facteurs individuels de variabilité PK des anticorps ont été aujourd'hui quantifiés, ils ne permettent, ensemble, d'expliquer qu'une partie de la variabilité et peuvent être insuffisants pour optimiser le schéma posologique. Pour les anti-TNF- $\alpha$, la posologie doit ainsi être adaptée à chaque patient [42]. L'existence d'une relation concentration-effet pour le bévacizumab [36] et le cétuximab [43, 44] plaident pour la nécessité d'un ajustement posologique fondé sur les concentrations. La modélisation PK est ainsi un outil de choix pour la détermination du schéma posologique optimal.[8] $\diamond$

\section{SUMMARY}

\section{Pharmacokinetic variability of therapeutic antibodies}

Therapeutic antibodies have been increasingly used for the treatment of various diseases, including cancers and chronic inflammatory diseases. The pharmacokinetic interindividual variability of mAbs is large and influences, at least in part, the clinical response to antibody treatment. This variability is explained by a number of individual sources of variability, which are reviewed here. Some of them are major because they are frequently reported to greatly influence the interindividual variability; notably, increased body size, the presence of antidrug antibodies, and high antigen mass are associated with decreased antibody concentrations. Other individual sources of variability are of less critical importance. They include sex, age, co-treatments, or genetic polymorphisms of IgG Fc receptors (Fc $\gamma R s$ ). The interindividual variability of antibody pharmacokinetics should be soundly described in order to design optimal dosing strategy. $\diamond$

\section{LIENS D'INTÉRÊT}

David Ternant a réalisé des formations pour les employés des laboratoires Amgen, Bohringer et Sanofi. Stéphanie Chhun déclare n'avoir aucun lien d'intérêt concernant les données publiées dans cet article.

\section{RÉFÉRENCES}

1. Dirks NL, Meibohm B. Population pharmacokinetics of therapeutic monoclonal antibodies. Clin $2010 ; 49: 633-59$

2. Dostalek M, Gardner I, Gurbaxani BM, et al. Pharmacokinetics, pharmacodynamics and physiologically-based pharmacokinetic modelling of monoclonal antibodies. Clin Pharmacokinet 2013; $52: 83-124$.

3. Ternant D, Bejan-Angoulvant T, Passot C, et al. Clinical pharmacokinetics and pharmacodynamics of monoclonal antibodies approved to treat rheumatoid arthritis. Clin Pharmacokinet 2015 ; 54 : 1107-23.

4. Ternant D, Karmiris K, Vermeire $S$, et al. Pharmacokinetics of adalimumab in Crohn's disease. EurJ Clin Pharmacol 2015 ; 71 : 1155-7.

5. Kagan L, Turner MR, Balu-lyer SV, Mager DE. Subcutaneous absorption of monoclonal antibodies: role of dose, site of injection, and injection volume on rituximab pharmacokinetics in rats. Pharm Res $2012 ; 29: 490-9$.

6. Lobo ED, Hansen RJ, Balthasar JP. Antibody pharmacokinetics and pharmacodynamics. J Pharm Sci 2004 ; 93 : 2645-68.

7. Paintaud G. Pharmacocinétique des anticorps monoclonaux. Med Sci (Paris) $2009 ; 25: 1057-62$.

8. Passot C, Pouw MF, Mulleman D, et al. Therapeutic drug monitoring of biopharmaceuticals may benefit from pharmacokinetic and pharmacokinetic-pharmacodynamic modeling. Ther Drug Monit $2017 ; 39: 322-6$.

9. Ternant D, Azzopardi N, Raoul W, et al. Influence of antigen mass on the pharmacokinetics of therapeutic antibodies in humans. Clin Pharmacokinet 2018 ; 2018 : 018-0680.

10. Ternant D, Paintaud G. Pharmacokinetics and concentration-effect relationships of therapeutic monoclonal antibodies and fusion proteins. Expert Opin Biol Ther 2005 ; 5 : S37-47.

11. Liu L. Pharmacokinetics of monoclonal antibodies and Fc-fusion proteins. Protein Cell $2018 ; 9$ h 15-32.

12. Yu T, Enioutina EY, Brunner HI, et al. Clinical pharmacokinetics and pharmacodynamics of biologic therapeutics for treatment of systemic lupus erythematosus. Clin Pharmacokinet 2017 ; 56 : 107-25.

13. Gill KL, Machavaram KK, Rose RH, Chetty M. Potential sources of inter-subject variability in monoclonal antibody pharmacokinetics. Clin Pharmacokinet $2016 ; 55: 789-805$.

14. Duffull SB, Wright DF, Winter HR. Interpreting population pharmacokineticpharmacodynamic analyses - a clinical viewpoint. Br J Clin Pharmacol $2011 ; 71: 807-14$.

15. Mould DR, Upton RN. Basic concepts in population modeling, simulation, and model-based drug development-part 2: introduction to pharmacokinetic modeling methods. CPT Pharmacometrics Syst Pharmacol $2013 ; 2013: 14$.

16. Fronton L, Pilari S, Huisinga W. Monoclonal antibody disposition: a simplified PBPK model and its implications for the derivation and interpretation of classical compartment models. J Pharmacokinet Pharmacodyn $2014 ; 41: 87-107$.

17. Gibiansky L, Gibiansky $\varepsilon$. Target-mediated drug disposition model: approximations, identifiability of model parameters and applications to the population pharmacokinetic-pharmacodynamic modeling of biologics. Expert Opin Drug Metab Toxicol 2009 ; 5 : 803-12. 


\section{RÉFÉRENCES}

18. Gibiansky L, Gibiansky $\varepsilon$, Kakkar T, Ma P. Approximations of the target-mediated drug disposition model and identifiability of model parameters. J Pharmacokinet Pharmacodyn $2008 ; 35: 573-91$

19. Baert F, Noman M, Vermeire $S$, et al. Influence of immunogenicity on the long-term efficacy of infliximab in Crohn's disease. $N$ EnglJ Med $2003 ; 348: 601-8$.

20. Radstake TR, Svenson M, Eijsbouts AM, et al. Formation of antibodies against infliximab and adalimumab strongly correlates with functional drug levels and clinical responses in rheumatoid arthritis. Ann Rheum Dis 2009 ; 68 : 1739-45.

21. Ternant $D$, Ducourau $\varepsilon$, Perdriger $A$, et al. Relationship between inflammation and infliximab pharmacokinetics in rheumatoid arthritis. Br J Clin Pharmacol $2014 ; 78: 118-28$.

22. Thurlings RM, Teng 0 , Vos $K$, et al. Clinical response, pharmacokinetics, development of human anti-chimaeric antibodies, and synovial tissue response to rituximab treatment in patients with rheumatoid arthritis. Ann Rheum Dis $2010 ; 69: 409-12$.

23. Kverneland $A H$, Enevold C, Donia M, et al. Development of anti-drug antibodies is associated with shortened survival in patients with metastatic melanoma treated with ipilimumab. Oncoimmunology $2018 ; 7: 2018$

24. Schmidt MM, Townson SA, Andreucci AJ, et al. Crystal structure of an HSA/FcRn complex reveals recycling by competitive mimicry of HSA ligands at a $\mathrm{pH}$-dependent hydrophobic interface. Structure $2013 ; 21: 1966-78$.

25. Lu JF, Bruno R, Eppler $S$, et al. Clinical pharmacokinetics of bevacizumab in patients with solid tumors. Cancer Chemother Pharmacol $2008 ; 62: 779-86$

26. Lixoft. Target-mediated drug disposition (TMDD) model library. Accessed at: http://mlxtran.lixoft.com/libraries/target-mediated-drug-disposition-tmdd-modellibrary/2017.

27. Tout $M$, Casasnovas 0 , Meignan $M$, et al. Rituximab exposure is influenced by baseline metabolic tumor volume and predicts outcome of DLBCL patients: a Lymphoma study association report. Blood 2017 ; 129 : 2616-23.

28. Ferri N, Bellosta S, Baldessin L, et al. Pharmacokinetics interactions of monoclonal antibodies. Pharmacol Res 2016; $111: 592-9$.

29. Djebli N, Martinez JM, Lohan L, et al. Target-mediated drug disposition population pharmacokinetics model of alirocumab in healthy volunteers and patients: pooled analysis of randomized phase I/II/III studies. Clin Pharmacokinet 2017 ; $56: 1155-71$.

30. Kuchimanchi M, Grover A, Emery MG, et al. Population pharmacokinetics and exposureresponse modeling and simulation for evolocumab in healthy volunteers and patients with hypercholesterolemia. J Pharmacokinet Pharmacodyn 2018 ; 2018: 018-9592.

31. Gibbs JP, Doshi S, Kuchimanchi M, et al. Impact of target-mediated elimination on the dose and regimen of Evolocumab, a human monoclonal antibody against proprotein convertase Subtilisin/ Kexin type 9 (PCSK9). J Clin Pharmacol 2017 ; 57 : 616-26.

32. Sun $H$, Van LM, Floch $D$, et al. Pharmacokinetics and pharmacodynamics of Canakinumab in patients with systemic juvenile idiopathic arthritis. J Clin Pharmacol 2016 ; 56 : 1516-27.

33. Malik P, Edginton A. Pediatric physiology in relation to the pharmacokinetics of monoclonal antibodies. Expert Opin Drug Metab Toxicol 2018 ; 14 : 585-99.

34. Ternant D, Arnoult C, Pugniere M, et al. IgGl Allotypes Influence the pharmacokinetics of therapeutic monoclonal antibodies through FcRn binding. J Immunol 2016 $196: 607-13$.

35. Sachs UJ, Socher I, Braeunlich CG, et al. A variable number of tandem repeats polymorphism influences the transcriptional activity of the neonatal Fc receptor alpha-chain promoter. Immunology $2006 ; 119: 83-9$.

36. Caulet $M$, Lecomte $T$, Bouche 0 , et al. Bevacizumab pharmacokinetics influence overall and progression-free survival in metastatic colorectal cancer patients. Clin Pharmacokinet 2016 ; 55 : 1381-94.

37. Passot C, Azzopardi N, Renault S, et al. Influence of FCGRT gene polymorphisms on pharmacokinetics of therapeutic antibodies. MAbs $2013 ; 5: 614-9$.

38. Dall'Ozzo S, Tartas S, Paintaud G, et al. Rituximab-dependent cytotoxicity by natural killer cells: influence of FCGR3A polymorphism on the concentrationeffect relationship. Cancer Res 2004 ; 64 : 4664-9.

39. Ternant D, Buchler M, Beneton M, et al. Interindividual variability in the concentration-effect relationship of antilymphocyte globulins: a possible influence of FcgammaRIlla genetic polymorphism. Br J Clin Pharmacol $2008 ; 65: 60-8$.

40. Ternant D, Buchler M, Thibault G, et al. Influence of FcgammaRIIIA genetic polymorphism on T-lymphocyte depletion induced by rabbit antithymocyte globulins in kidney transplant patients. Pharmacogenet Genomics $2014 ; 24: 26-34$

41. Sharma S, Mittapalli RK, Holen KD, Xiong H. Population pharmacokinetics of ABT-806, an investigational anti-epidermal growth factor receptor (EGFR) monoclonal antibody, in advanced solid tumor types likely to either over-express wild-type EGFR or express variant III mutant EGFR. Clin Pharmacokinet $2015 ; 54: 1071-81$.

42. Paintaud G, Passot C, Ternant D, et al. Rationale for therapeutic drug monitoring of biopharmaceuticals in inflammatory diseases. Ther Drug Monit 2017 ; 39 : 339-43.

43. Azzopardi N, Lecomte T, Ternant D, et al. Cetuximab pharmacokinetics influences progression-free survival of metastatic colorectal cance patients. Clin Cancer Res 2011 ; 17 : 6329-37.

44. Pointreau Y, Azzopardi N, Ternant D, et al. Cetuximab pharmacokinetics influences overall survival in patients with head and neck cancer. Ther Drug Monit 2017 ; 38 : 567-72.

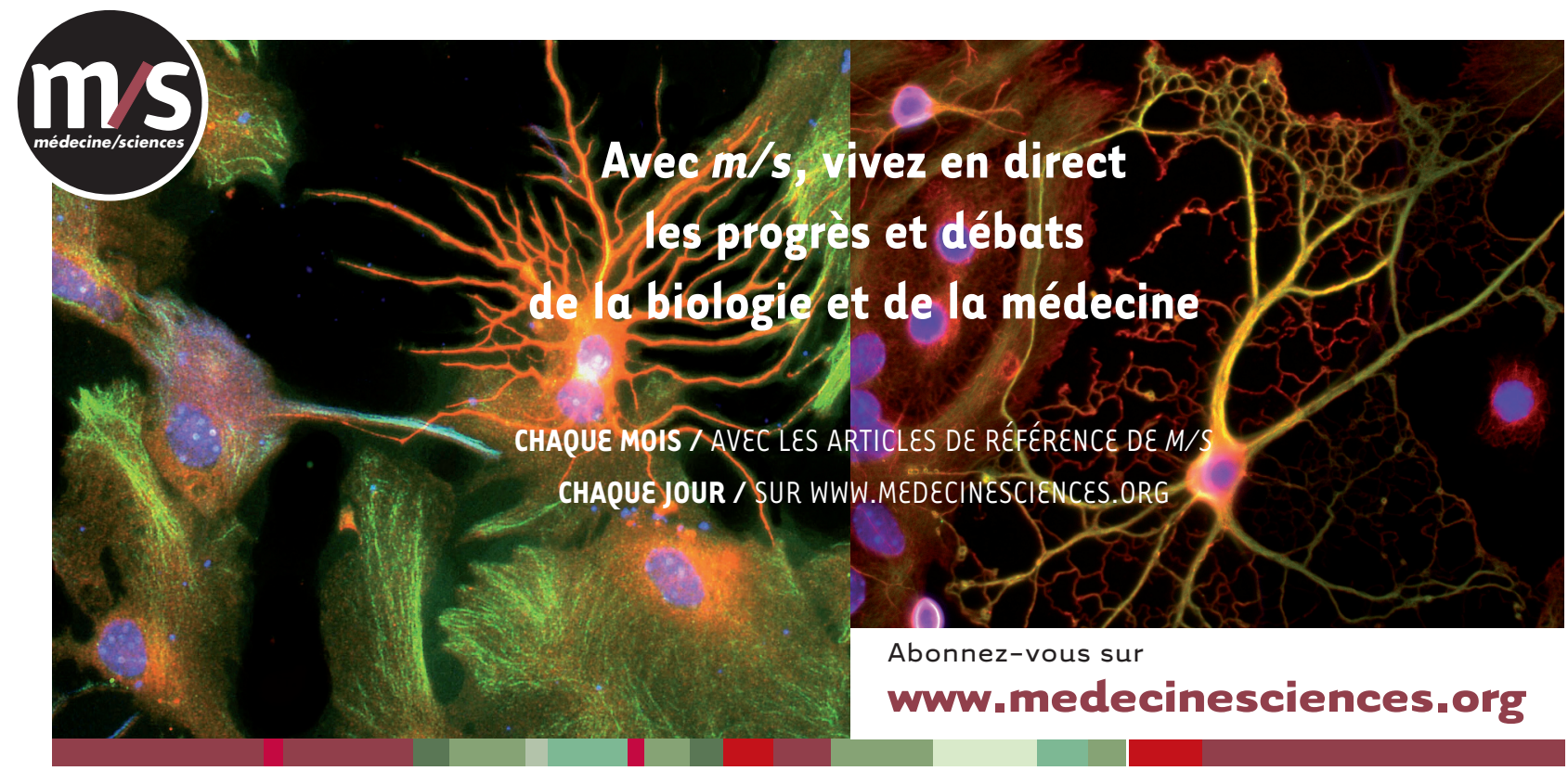

TIRÉS À PART

D. Ternant 